\title{
Desabastecimiento de medicamentos esenciales en las unidades médicas de la red pública integral de salud en Ecuador
}

\section{Shortage of essential medicines in the medical units of the comprehensive public health network in Ecuador}

\author{
MSc, Arcadio Jacinto Giacoman Jiménez ${ }^{1}$ \\ agiacomanj@iess.gob.ec \\ PhD, Milton Rafael Maridueña Arroyave ${ }^{2}$ \\ milton.mariduenaa@ug.edu.ec
}

Recibido: 1/04/2019; Aceptado: 1/06/2019

\begin{abstract}
RESUMEN
El Instituto Suramericano de Gobierno en Salud (ISAGS) ha presentado una investigación desarrollada con datos de los Ministerios de Salud de la región, sobre el riesgo de desabastecimiento de medicamentos esenciales, ha informado la institución. La especialista en medicamentos y tecnologías de la salud del ISAGS, Ángela Acosta, en colaboración con el Organismo Andino de Salud - Convenio Hipólito Unanue (ORAS- CONHU) han presentado el texto "Situación de Medicamentos Esenciales con Riesgo de Desabastecimiento con Énfasis en los Países Suramericanos". En la investigación, tras una descripción documentada de la situación de desabastecimiento de medicamentos esenciales en el ámbito regional y global, fueron identificados las causas, frecuencia, impacto y medicamentos afectados por el problema. El listado de medicamentos desabastecidos ha sido elaborado con los datos de los Ministerios de Salud de la región, pero la publicación incluye posibles estrategias de abordaje, así como indicadores de manejo e impacto.
\end{abstract}

Palabras Clave: desabastecimiento, esenciales, estrategias

\footnotetext{
${ }^{1}$ Universidad de Guayaquil, Guayaquil, Ecuador.

2 Universidad de Guayaquil, Guayaquil, Ecuador.
} 


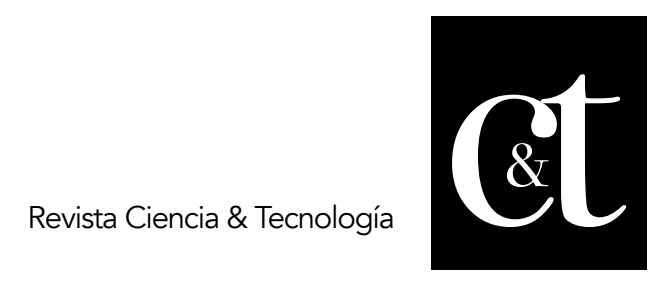

No. 23, 31 de julio de 2019

ISSN impreso: 1390 - 6321

ISSN online: 2661 - 6734

\begin{abstract}
The South American Institute of Government in Health (ISAGS) has presented an investigation developed with data from the Ministries of Health of the region, on the risk of shortage of essential medicines, the institution has informed. The specialist in medicines and health technologies of ISAGS, Angela Acosta, in collaboration with the Andean Health Organization - Hipolito Unanue Agreement (ORAS-CONHU) has presented the text "Situation of Essential Drugs at Risk of Shortages with Emphasis on Countries South American". In the investigation, after a documented description of the situation of shortages of essential medicines in the regional and global scope, the causes, frequency, impact and drugs affected by the problem were identified. The list of medicines that have not been supplied has been prepared with data from the Ministries of Health of the region, but the publication includes possible strategies for addressing them, as well as management and impact indicators.
\end{abstract}

Keywords: shortage, essential, strategies

\title{
Introducción
}

La Red Pública Integral de Salud es la unión o conjunto organizado, de las Instituciones Públicas para prestar un continuo, coordinado e integral servicio de salud a la población ecuatoriana. El objetivo de la Red Pública es coordinar la formulación de propuestas de políticas públicas para la gestión del suministro de medicamentos y dispositivos médicos y coordinar el desarrollo de estrategias para mejorar la logística y suministro de medicamentos y dispositivos médicos en la Red pública Integral de Salud.

Entre sus funciones están: incrementar la promoción del uso racional de medicamentos a nivel nacional mediante la generación e implementación de un programa de difusión; construir el Sistema Nacional de Salud, que permita garantizar el acceso universal de los ecuatorianos a los servicios públicos de salud, estructurados en una red pública y con atenciones de calidad, eficiencia y sin costo para el usuario. La implementación de la red pública integral es parte del proyecto nacional que busca transformar el sector salud, fortaleciendo lo público con miras a lograr la universalidad de la salud y la gratuidad de los servicios en el punto de recepción del servicio está conformado por MSP, IESS, ISSPOL, ISSFA dentro esta conformación el Gobierno nacional mediante normas y estatutos dispuso al SERCOP que el $90 \%$ de los medicamentos ingresen en el cuadro básico.

Se realizan compras mediante un sistema compra mediante el catálogo electrónico. Una de la ventajas es el menor precios el mercado; pero desventajas en medicamento genéricos y mediante convenio marco estipula solo tres casas fabricantes para toda producción nacional. Adicionalmente, los proveedores siguen entregando con fechas mayores a la interpuesta en convenio marco y son reportados a nivel nacional. Pero, otro problema es el que se genera al paciente, quien no tiene acceso a los medicamentos al momento dela prescripción del médico tratante. Las unidades de la RPIS en Ecuador sufren actualmente un desabastecimiento y se han 


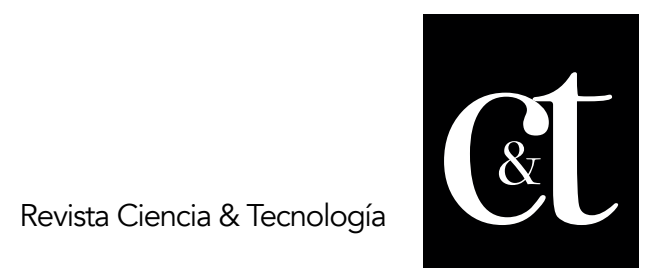

No. 23, 31 de julio de 2019

ISSN impreso: 1390 - 6321

ISSN online: 2661 - 6734

buscado otros mecanismos para mantener el abastecimiento, pero no se puede llegar a un indicador alto y adecuado para evitar los stocks críticos.

Coherentes con la transformación sectorial de la salud en el Ecuador, cuyo objetivo es "garantizar el derecho de la salud para todos y todas" y de acuerdo con la Constitución de la República vigente, que favorece la transición hacia un modelo de salud pública, integral, de acceso universal, equitativo y sin costo directo para el usuario"1, es necesario la participación de los diversos actores del Estado de manera que se logre contribuir al desarrollo de una sociedad ecuatoriana más saludable.

\section{Desarrollo}

El no cumplimiento de entrega de medicinas a las unidades médicas por los proveedores incumplidos sigue trayendo resultados no positivos, ya que las unidades RPIS están en déficit del 30 al $40 \%$ de desabastecimiento. Esto fundamenta una mala norma y ley de contratación y elección, con solo tres casas comerciales farmacéuticas que traen o alucen el riesgo de las autoridades en sus cargos por desabastecimientos, ya que deberían abrir el abanico de dispersión a otras empresas de menor, mediana y mayor producción para la entrega de los fármacos o buscar mecanismo alternos con viabilidad de las autoridades nacionales ya que traen un perjuicio a los pacientes con patologías crónicas y evolución agudas, catastróficas con riesgo alto de morbilidad y mortalidad al no constar este principio activo en los pacientes. Ellos realizan denuncias por el desabastecimiento.

Los elementos que se consideran la normativa para adquisición de fármacos son (LOSCP, 2008):

Art. 43.-Convenios Marco.-El Servicio Nacional de Contratación Pública efectuará Periódicamente procesos de selección de proveedores con quienes se celebrará Convenios Marco en virtud de los cuales se ofertarán en el catálogo electrónico Bienes y servicios normalizados a fin de que éstos sean adquiridos o contratados de manera directa por las Entidades Contratantes, sobre la base de parámetros y objetivos establecidos en la normativa que para el efecto dicte el Instituto Nacional de Contratación Pública.

Art. 44.-Catálogo Electrónico. - Como producto del Convenio Marco, el Servicio Nacional de Contratación Pública creará un catálogo electrónico disponible en el Portal COMPRAS PÚBLICAS, desde el cual las Entidades Contratantes podrán realizar sus adquisiciones en forma directa.

Art. 45.-Obligaciones de los Proveedores. - Los adjudicatarios quedarán obligados a proveer bienes y servicios normalizados de conformidad con las condiciones de plazo, precio, calidad, lugar de entrega y garantía establecidas para el período de duración del Convenio Marco. No obstante, los adjudicatarios podrán mejorar las condiciones establecidas, siguiendo el procedimiento que para el efecto se haya previsto en el Convenio Marco.

Art. 46.-Obligaciones de las Entidades Contratantes. -Las Entidades Contratantes deberán consultar el catálogo electrónico previamente a establecer procesos de adquisición de bienes y servicios. Si el bien o servicio requerido no se encuentre catalogado, se podrán realizar otros procedimientos de selección para la adquisición de bienes o servicios, de conformidad con la presente Ley y su Reglamento. 


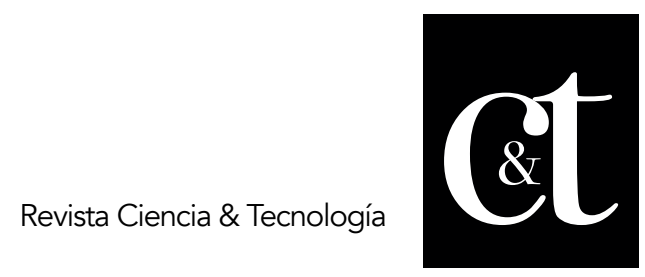

No. 23, 31 de julio de 2019

ISSN impreso: 1390 - 6321

ISSN online: 2661 - 6734

Si cualquiera de las entidades contratantes obtuviere ofertas de mejor costo que las que consten publicadas en el catálogo electrónico, deberán informar al Servicio Nacional de Contratación Pública para que éste conozca y confirme que la oferta es mejor y adopte las medidas necesarias que permitan extender tales costos, mediante la celebración de Convenios Marco, al resto de Entidades Contratantes.

Art. 69.-Suscripción de Contratos. - Los contratos que por su naturaleza o expreso mandato de la Ley lo requieran se formalizarán en escritura pública dentro del término de quince (15) días desde la notificación de la adjudicación. Los contratos cuya cuantía sea igual o superior a la base prevista para la licitación se protocolizarán ante Notario Público. Los gastos derivados del otorgamiento del contrato son de cuenta del contratista. Las contrataciones que se realicen por el sistema de catálogo se formalizarán con la orden de compra y el acta de entrega.

\section{Artículos relacionados en el RGLOSNCP}

Art. 16.-Micro, pequeño y medianas empresas. - Para incentivar la mayor participación de proveedores de los sectores de micro, pequeñas y medianas empresas -MIPYIMES, se entenderán por tales, aquellas que cumplan los parámetros establecidos de conformidad con el artículo 53 del Código Orgánico de la Producción, Comercio e Inversiones.

Al momento de inscribir y habilitar a un proveedor en el RUP, el registro deberá expresar la categoría a la que pertenece el proveedor.

EI INCOP establecerá criterios de preferencia a favor de las MIPYMES, a través de alguno de los siguientes mecanismos:

1. Márgenes de preferencia sobre las ofertas de otros proveedores;

2. Criterios para contratación preferente establecidos en el artículo 52 de la Ley;

3. Siempre que, luego de las evaluaciones de ofertas, exista la posibilidad de adjudicar a una MIPYME y a otro proveedor que no tenga esta calidad, se preferirá a aquella;

4. Posibilidad de que las MIPYMES mejoren su propuesta para que puedan igualar o superar la oferta de otros proveedores, luego de la evaluación de ofertas.

5. Inclusión, en el catálogo electrónico, de bienes o servicios provenientes de MIPYMES, artesanos o actores de la economía popular y solidaria para que sean adquiridos preferentemente por las entidades contratantes. El procedimiento de selección a emplearse por parte del INCOP para esta catalogación será la feria inclusiva.

Las preferencias para los micros, pequeñas y medianas empresas se aplicarán en función de que su oferta se catalogue como de origen nacional, por el componente nacional que empleen, de tal manera que no se otorgarán estos beneficios a meros intermediarios. Los beneficiarios a favor de las MIPYMES se harán extensivos a actores de la economía popular y solidaria, de conformidad con la ley.

Art. 43.-Procedimiento para contratar por catálogo electrónico. -Para la inclusión en 


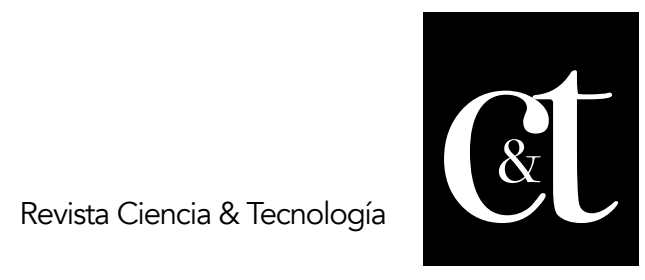

No. 23, 31 de julio de 2019

ISSN impreso: 1390 - 6321

ISSN online: 2661 - 6734

el catálogo electrónico de los bienes y servicios normalizados, el INCOP realizará procesos de selección que permitan celebrar convenios marcos, observando el procedimiento que se establezca en los pliegos. Las contrataciones por catálogo electrónico de bienes y servicios normalizados, que realicen las Entidades Contratantes, observarán el procedimiento señalado por el INCOP.

La orden de adquisición electrónica emitida por la Entidad Contratante se sujetará a las condiciones contractuales previstas en el Convenio Marco; y, de ser el caso a las mejoras obtenidas por la entidad contratante. De conformidad con lo previsto en el inciso segundo del artículo 69 de la Ley, la Orden de Compra emitida a través del Catálogo Electrónico formaliza la contratación de los bienes o servicios requeridos y genera los derechos y obligaciones correspondientes para las partes.

Una vez recibidos los bienes o servicios contratados, se suscribirá el acta de entrega recepción correspondiente con la verificación de correspondencia con las especificaciones previstas en el catálogo.

\section{Conclusiones}

En cuanto a la no entrega oportuna post generación de ordenes catalogó electrónico a la compra de medicamentos esenciales para RPIS (red pública Integral de Salud en Ecuador) esto traen como resultado desabastecimiento principales medicamentos para los tratamientos de enfermedades crónicas y agudas que diariamente acude a nuestras consultas médicas y además incumplimiento de proveedores asignados por el SERCOP mediantes sanciones pecuniarias por cada entidad de salud y notificado por su incumplimiento pero sigue continuando al no abrir el abanico comercial tanto por mayorista fabricantes y distribuidores que puedan abastecer a nuestra red de salud es prioritario que las Autoridades actúen de manera ipso facto en aplicar nuestra estrategias y leyes para implementar plan de contingencia evitar esta novedades urgencia por la causa enunciada este articulo para mejorar las necesidades y tener el abastecimiento oportuno y real en cada unidad médica del Ecuador

\section{Referencias bibliográficas}

Ministerio de Salud Pública, «instituciones.msp.gob.ec,» 2008. [En línea] Recuperado de:

http://instituciones.msp.gob.ec/dps/morona_santiago/index.php?option=com_con tent\& $\mathrm{v}$ iew $=$ article\&id=157: rpis\&catid=12: programas\&Itemid $=10$.

Ministerio de Salud Pública, «Salud.gob.ec,» Diciembre 2009. [En línea].

Available: https://www.salud.gob.ec/wp-content/uploads/2017/03/0915-A.ministerial-manual-de-procesos-para-la-gestión-de- medicamentos-en-el-msp-2812-2009.pdf.

SERCOP (Portal.compraspublicas.gob.ec) Enero 2015.

Descargado de: https://portal.compraspublicas.gob.ec/sercop/wpcontent/uploads/downloads/2015/09/Manual-SOCE-Compra-por-catalogoRepertorio-de-Medicamentos-Proveedores.pdf. 